\title{
Las cooperativas de crédito y la financiación de los sistemas productivos locales: el caso del sistema productivo agroindustrial de Almería
}

\author{
Credit unions and financing of local production systems: the case of the agro industrial \\ production system of Almería
}

\section{As cooperativas de crédito e o financiamento dos sistemas produtivoslocais: o caso do sistema produtivo agroindustrial de Almería}

Luis Jesús Belmonte-Ureña ${ }^{1}$, Francisco Joaquín Cortés-García ${ }^{2}$, Valentín Molina-Moreno ${ }^{3}$

Forma de citar: L.J. Belmonte-Ureña, F.J. Cortés-García y V. Molina-Moreno "Las cooperativas de crédito y la financiación de los sistemas productivos locales: el caso del sistema productivo agroindustrial de Almería", Respuestas, vol. 22, no. 1, pp. 48-58, 2017.

Recibido:

Mayo 30 de 2016

Aceptado:

Octubre 19 de 2016

\section{Resumen}

El objetivo de este trabajo consiste en analizar la correlación que existe entre el modelo productivo de base agrícola de Almería y la cooperativa de crédito más importante de España, Cajamar Caja Rural, por estar estrechamente vinculados desde su origen y haber constituido un complejo sistema productivo local. Para ello, se parte de una breve descripción del modelo de desarrollo local de Almería, de sus ventajas y de sus amenazas. Asimismo, se apunta la oportunidad de estudiar este modelo, de cara a la implantación en otras regiones o países, tras analizar la bondad de su aplicación. Posteriormente, se describen las fases de financiación del crecimiento, de base cooperativa, detectándose un notable desfase entre la inversión crediticia de Almería y el volumen de depósitos captados a otros sectores residentes. Para concluir, se presenta un apartado con las principales reflexiones de este trabajo, entre las que destaca la necesaria relación simbiótica entre el sector de cooperativas de crédito y el modelo productivo almeriense.

Palabras clave: Almería, Cajamar Caja Rural, cooperativas de crédito, modelo de desarrollo agrícola.
Abstract:
The aim of this paper is to analyze the correlation between the production model of agricultural base of Almeria and the most important credit union in Spain, Cajamar Caja Rural, by being closely linked from the beginning and had been a complex local productive system . To do this, we start from a brief description of the local development of Almeria, its advantages and its threats. Also, the opportunity to study this model is pointed out, in order to implement it in other regions or countries, after analyzing the benefits of its application. Later, the phases of financing of the growth, cooperative basis are described, detecting a significant gap between Almeria loans and the volume of deposits attracted to other residents. In conclusion, a section with the main thoughts of this work is presented, emphasizing the necessary symbiotic relationship between the credit unions sector and the production model from Almería. 
Luis Jesús Belmonte-Ureña, Francisco Joaquín Cortés-García, Valentín Molina-Moreno

Keywords: Almería, Cajamar Caja Rural, credit union, agricultural development model

\section{Resumo}

O objetivo deste trabalho consistiu em analisar a correlação que existe entre o modelo produtivo de base agrícola de Almería e a cooperativa de crédito mais importante da Espanha, Cajamar Caixa Rural, por estar estreitamente vinculados desde sua origem e ter constituído um complexo sistema produtivo local. Para fazer isso, partimos de uma breve descrição do modelo de desenvolvimento local de Almería, de suas vantagens e de suas ameaças. Assim mesmo, se apontou a oportunidade de estudar este modelo, em face da aplicação em outras regiões ou países, depois de analisar a bondade de seu aproveitamento. Posteriormente, foram descritas as fases de financiamento do crescimento, de base cooperativa, detectando-se um atraso perceptível entre a inversão creditícia de Almería e o volume de depósitos recebidos a outros sectores residentes. Em conclusão, apresentou-se uma seção com as principais reflexões deste estudo, destacando-se a necessária relação simbiótica entre o sector de cooperativas de crédito e o modelo produtivo almeriense.

Palavras-chave: Almería, Cajamar Caixa Rural, cooperativas de crédito, modelo dedesenvolvimento agrícola.

\section{Introducción}

El modelo de crecimiento del sureste español, basado en la producción hortofrutícola, lleva años despertando el interés de numerosos países en vías de desarrollo y con un perfil muy específico [1], [2], [3]. El perfil de estos países, a los que se les podría aplicar el modelo de crecimiento agroindustrial de Almería, con base en empresas agropecuarias y, fundamentalmente, en economía social, se caracteriza por:

- La existencia de un mix productivo poco diversificado, con especialización en un único sector productivo, normalmente primario. Esto implica la inexistencia de un sector industrial que apoye el despegue económico, y mucho menos un sector terciario relevante.

- Poseer un sector agrícola de bajo rendimiento y en el que la aplicación de un modelo intensivo basado en el crecimiento de la productividad, con tecnología de invernaderos y optimización de agua, podría generar un crecimiento sostenido y un alto nivel de empleo, desde el punto de vista laboral.

- Carecen de sólidas estructuras de financiación de la actividad productiva, entre otras cosas por la alta tasa de informalidad de sus economías, por la dificultad de acceder a los mercados internacionales y por la escasa fortaleza de su sector financiero. A este respecto, un modelo de economía social, aplicado al sector agrícola intensivo requiere un moderado nivel de capitalización, pues el volumen de inversión en infraestructuras y el apalancamiento necesario no son elevados.

Llegados a este punto, hay que explicar en qué consiste el modelo de agricultura intensiva almeriense. En este sentido, hay que reconocer la amplia trayectoria con la que cuenta, pues 
ser remonta a los años 60, convirtiéndose en el sector estratégico de la provincia almeriense, seguido por la aportación del sector turístico y la explotación de las canteras de mármol.

Hoy por hoy, el referente económico de esta provincia se encuentra en la agricultura intensiva bajo invernadero y en el entramado empresarial, de base cooperativa, que se ha generado a su alrededor. Su éxito durante tanto tiempo y su capacidad exportadora hacen pensar en la alternativa y el ejemplo que este modelo ofrece para otros países. Efectivamente, se puede afirmar que la agricultura almeriense es la más productiva de España, por su intensidad en la inversión en tecnología aplicada a los cultivos intensivos, lo que le ha reportado tasas de productividad de índole cuasi industrial [4], [5].

La pregunta que cabe hacerse ahora es: ¿Cuáles son los condicionantes que han posibilitado la creación de este sistema productivo agrario de alto rendimiento? A este respecto, parece obvio que la existencia de este modelo ha requerido de ciertos condicionantes que han favorecido su implantación y desarrollo.

A partir de ahora el trabajo continúa con la exposición del modelo de desarrollo local agroindustrial de la provincia de Almería, en el que se detalla el perfil del modelo así como las amenazas que se ciernen sobre él. Posteriormente, en el tercer apartado, se exponen las fases del desarrollo de la financiación de este modelo productivo, que creció paralelo al desarrollo de la agricultura intensiva de Almería. Para finalizar, se presenta un apartado con las principales conclusiones de este trabajo.

\section{EI modelo de desarrollo local agroindustrial de Almería.}

En poco menos de medio siglo, Almería ha pasado de ser una provincia deprimida, económicay demográficamente hablando,para convertirse en una provincia especialmente dinámica. Sin embargo, es cierto que tendrá que abordar cambios sustanciales en la estructura de las explotaciones, pues tendrá que orientarse hacia un modelo de calado industrial, con base agrícola.

Hasta ahora, hay que reconocer que el sistema productivo almeriense se ha convertido en un modelo agronómico endógeno de esta provincia, que ha basado su éxito en el saber hacer de sus agricultores, en la intensidad en el uso de tecnología, artes y conocimiento, que han supuesto la reutilización de tierras que antaño eran baldías, gracias a los nuevos usos del agua y la tecnología de invernaderos. No olvidemos que, existen marcos globales de gestión que buscan obtener las ventajas mediante la diferenciación de productos, compitiendo básicamente con el concepto de gestión de la calidad [6]

En la figura 1 se presenta la relevancia de cada sector productivo en el conjunto del valor añadido bruto nacional. De los tres ámbitos geográficos considerados, Almería, Andalucía y España, resulta que la provincia almeriense es la que mayor peso del sector agrícola ostenta (13\%), sobre el total generado por los tres sectores productivos. Esta evidencia da una idea sobre la importancia del modelo de desarrollo local, de base agroalimentaria, en la provincia, pues más que duplica el porcentaje que alcanza la media andaluza (5\%) y más que cuadriplica al 3\% de la media nacional. 


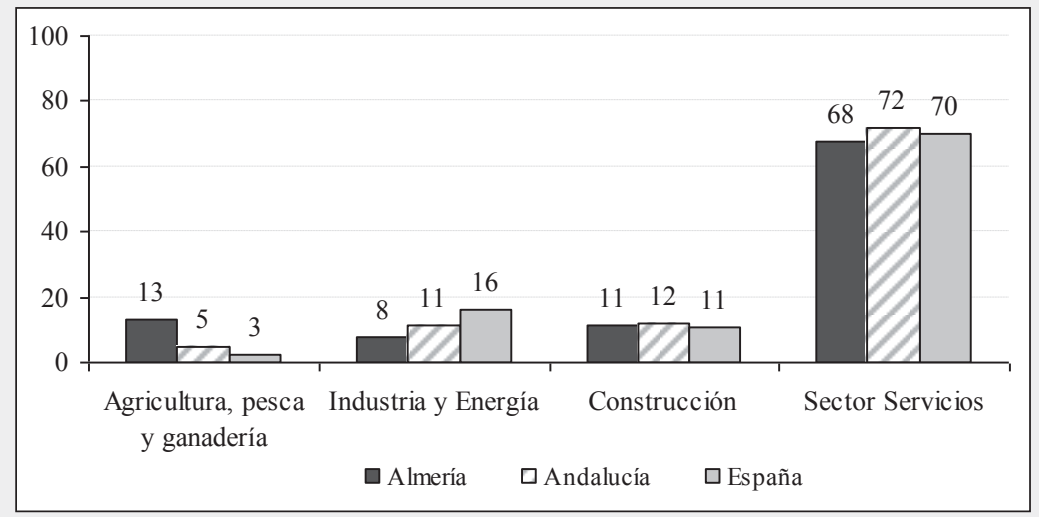

Enero - Junio 2017

ISSN 0122-820X

E-ISSN 2422-5053

Figura 1. Distribución sectorial del V.A.B. (2010) Fuente: elaboración propia a partir de [7]

En 2008, antes de presenciar los efectos más adversos de la crisis, los porcentajes de representatividad del V.A.B del sector primario eran: $12 \%, 5 \%$ y $3 \%$, para Almería, Andalucía y España, respectivamente. A este respecto, el advenimiento de la crisis ha convertido al sector agrícola de la provincia en un refugio contra la contracción de la economía.

En cuanto al peso agrícola de la provincia de Almería, los datos suministrado por la Contabilidad Regional de España, indican que la provincia representa más del $6 \%$ de valor añadido bruto del sector primario (1.496 millones de euros de Almería, frente a los 24.554 millones de euros de España). Sin embargo, esa representatividad que ostenta esta provincia sobre el total nacional, en cuanto a V.A.B se refiere apenas supera el 1\%, tras agregar todos los sectores productivos.

Así pues, ¿con qué características cuenta el modelo de desarrollo local agroindustrial de Almería para presentar esa representatividad? Se pueden identificar las siguientes:

- Se trata de un sistema productivo que se circunscribe a unas fronteras geográficas muy concretas, en las que se aplica un saber hacer y una forma de producir muy homogéneas, localizado en el sureste español.
- Es un modelo endógeno con orientación comercial hacia el exterior, es decir, los parámetros de su crecimiento aprovechan los recursos locales (trabajo y capital) para orientar sus producciones a la exportación (Con del despegue del modelo productivo almeriense, pronto se necesitó exportar la mayor parte de la producción. En este sentido, en primera instancia, se recurrió a los grandes países de la Europa central , para pasar a los países del Este, repúblicas de la extinta URSS, Latinoamérica, Estados Unidos y Canadá [8]). En este sentido, la naturaleza de las rentas en el sector agrario han tenido esencialmente un carácter mixto, capital familiar y trabajo.

- Su competitividad se basa en la existencia de economías de escala externas, es decir, sus ganancias de eficiencia no surgen prioritariamente del ahorro en los costes internos de la empresa, a medida que se aumenta la producción. Su mayor ventaja está en las ganancias que se producen cuando los costes de ciertos servicios, como el transporte, la logística de semilleros, fitosanitarios, plástico y aceros, se reducen drásticamente a medida que el sistema productivo gana dimensión.

Según [9], las economías de escala externas se generan progresivamente a medida que se concentran pequeñas explotaciones, como 
es el caso de Almería, y generan servicios mayoristas para ciertos abastecimientos, a la vez que reducen su coste (descuentos en la adquisición de inputs, optimización de los sistemas de gestión/producción, aprendizaje colaborativo, etc.).

- Es un modelo alternativo y, la mayoría de las veces, soporte de un incipiente sector industrial, auxiliar de la agricultura, con el que combatir el desfase industrial que pueden tener las economías emergentes.

- Desde el punto de vista productivo, es un modelo de producción fácilmente exportable, que favorecerá la convergencia con los países más industrializados, pues comienza con una especialización en la producción agraria, en la que se optimiza la utilización de factores productivos, para llegar a un modelo intraindustrial mucho más complejo.

- Existencia de un modelo de financiación de base cooperativa. En el caso concreto de Almería, no se puede hablar de un modelo de desarrollo local genuino sin tener en cuenta el apoyo financiero, incondicional, que ha recibido por parte del sector de cooperativas de crédito, que comparte base social con las cooperativas agrícolas locales,generando ciertas descompensaciones en cooperativas que han realizado importantes inversiones en tecnología [10],[6]. Sin embargo, no se puede hablar de un auténtico modelo de financiación, por varias razones:

- Por la excesiva dependencia de los servicios financieros que ofrece la única cooperativa de crédito de Almería: Cajamar Caja Rural, lo que ha impedido que se generen secciones de crédito dentro del importante sector de cooperativas agrícolas almeriense, que sí cuenta con un alto nivel de competencia.
- Porque el sector agroalimentario almeriense no ha generado suficientes recursos económicos para financiar su actividad, por lo que su crecimiento se ha sufragado con el ahorro de otras provincias. Esta es una de las razones que llevó a Cajamar Caja Rural, a iniciar un importante proceso de expansión que la han convertido en la cooperativa de crédito más importante del país y en la décimo secta entidad más importante del sistema financiero español [11].

No obstante, el modelo de desarrollo local agroalimentario almeriense se enfrenta avarias amenazas, que tendrá que disipar en un futuro próximo. De manera esquemática, los riesgos a los que se enfrenta son:

- Aunque se trata de modelo maduro y probado durante varias décadas, está rozando los límites de la productividad por hectárea de terreno y rendimientos de la mano de la mano de obra. Efectivamente, en la actualidad este modelo se encuentra en el límite operativo de capitalización, es decir, de sustitución de mano de obra por tecnología, y en el límite de creación de empleo. Es por ello que el sector precisa que se aborde un verdadero plan estratégico que aborde el problema de la renovación de las estructuras agrícolas, las necesidades de capitalización del sector, la concentración del mismo con objeto de abordar proyectos de inversión más amplios, así como la racionalización de los costes y la mejora de la calidad del producto [12].

- Comercialmente, se ha perdido la ventaja comparativa que atesoraba la agricultura intensiva española con respecto a terceros países competidores, como es el caso de Marruecos, Turquía e Israel[13]. Además, la estrategia de comercialización actual está orientada hacia la venta intensiva, 
es decir, hacia la colocación de la mercancía que periódicamente deposita el productor en su cooperativa/alhóndiga que ejerce como comercializadora. A su vez, las comercializadoras son de escasa dimensión, por lo que no adquieren objetivos estratégicos propios al margen de los intereses de los intereses del socio/ productor. Asimismo buscando nuevos canales de comercialización del producto y sus derivados con menor dependencia de intermediarios internacionales [14].

- Limitaciones físicas (superficie cultivable), medioambientales (pesticidas y reciclaje de desechos de la horticultura) y de infraestructura (comunicaciones, hidrológicas, etc). Este hecho puede suponer un problema a la hora de enfrentar los desequilibrios que generará en el futuro.

Finalmente, se podría pensar en la extensión de la bondad del modelo almeriense, es decir, la implantación en otros territorios. Sin embargo, se plantea una duda importante, pues aunque está demostrada su eficacia en el sureste español, no se conoce cuál puede ser el resultado en todo un país, como tampoco se sabe si servirá de base a todo un sistema productivo. No obstante, en la última década, el saber hacer de varios cooperativistas almerienses ha traspasado sus fronteras y se ha comenzado a deslocalizarse, no sólo la comercialización de hortalizas[15], [9], sino también las estructuras productivas[16],[17], [18].

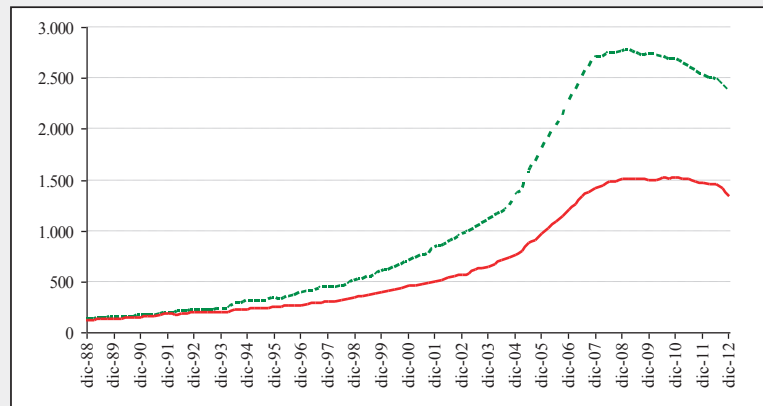

(a)

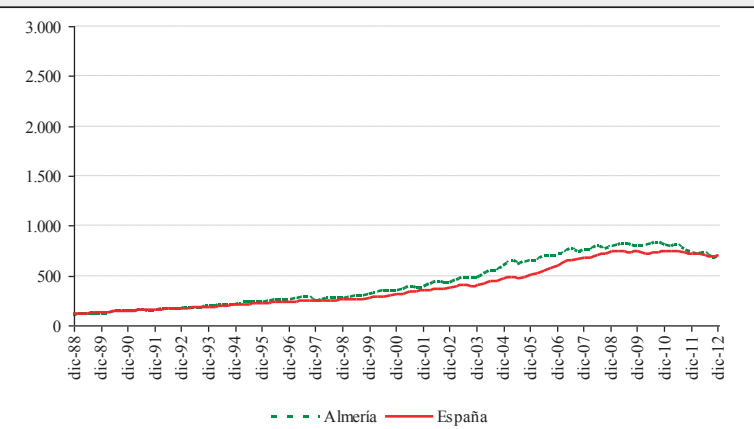

\section{La financiación del sistema productivo local de Almería.}

Desde finales de los 60, cuando comenzó a vislumbrarse el potencial del modelo productivo agrícola almeriense, también comenzaron a desarrollarse mecanismos de financiación ad hoc, acordes al perfil del incipiente sector productivo. Se trata pues de un sistema de financiación que surgió con el propio modelo productivo almeriense $\mathrm{y}$ que hoy por hoy es muy difícil de desligar uno del otro, o separar la causa del efecto. Ambos sectores, el financiero y el productivo, han crecido juntos, pues previamente no existía ninguna estructura de financiación capaz de acompañar el sector productivo almeriense[12]. Sin embargo, no se puede pensar en que el sector productivo generase los recursos financieros suficientes para sustentar el ritmo inversor que registró la provincia de Almería.

En los últimos 25 años, la expansión del crédito en la provincia de Almería ha superado ampliamente a la media nacional. Tal y como se muestra en la figura 2 . Se trata de un reflejo inequívoco de la necesidad de financiación con la que suportar la expansión de esta economía provincial, que alcanzó su máximo en 2007, es decir, el año en que comenzase la vislumbrarse el agotamiento del modelo de crecimiento español: basado en la construcción, especialmente a partir de 2004. 
Por su parte, según los datos suministrados por el Banco de España, el volumen de depósitos ha sido muy deficiente, tanto en España como en Almería, demostrándose insuficientes para financiar la expansión económica.

Así pues, el problema siempre fue el mismo y estuvo sobre la mesa: ¿cómo financiar la actividad productiva en una región sin ahorro? Para encontrar la respuesta más satisfactoria, a continuación se presentan las fases por las que ha pasado la conformación del actual modelo de financiación del modelo de desarrollo local almeriense:

- Fase inicial de escasa vinculación entre el sector financiero y el productivo- de la transición al Mercado Común (1960 a 1986). Se trata de una fase en la que las necesidades de financiación del campo eran escasas, pues en la fase de "colonización" del campo almeriense, el objetivo era la agricultura de subsistencia [20], [21]. La falta de alternativas productivas hizo que los primeros agricultores almerienses sólo tuvieran una opción real: la de perseverar en el desarrollo agrícola de su territorio.

En esta fase no surgió ninguna iniciativa sólida de desarrollar las secciones de crédito de las cooperativas agrícolas. Así, la financiación al campo se instrumentaba a través de los préstamos de campaña que concedían las alhóndigas.

- Fase de despegue económico - de ingreso y plena implantación del mercado único (1986 a 1993). En esta etapa se alcanza la vinculación del sector agrícola y el compromiso financiero de una entidad, de tipo cooperativo, como fue Caja Rural de Almería, hoy Cajamar Caja Rural. En esta fase se asienta el futuro desarrollo del modelo productivo almeriense. Sin embargo, en esta fase aún persistía la escasez de recursos financieros autóctonos con los que financiar la inversión que requería esta provincia, por lo que la sustitución de trabajo por medios de capital se hacía muy lentamente.

Es en esta fase cuando Caja Rural de Almería, ahora Cajamar Caja Rural, inicia su estrategia de expansión por otras provincias españolas, en las que captar el ahorro suficiente para financiar el crecimiento provincial, por lo que ya resultaba innecesaria la maduración de las secciones de crédito de las cooperativas agrícolas.

- Fase de madurez - de crecimiento e internacionalización (1993 a 2012). En esta etapa se produce la consolidación definitiva de Cajamar Caja Rural, como entidad financiera que financia con carácter preferente y, a veces, exclusivo al campo almeriense. En este sentido, la ganancia de dimensión de esta cooperativa de crédito, consolidada con sucesivos procesos de fusión con otras entidades cooperativas, como con una activa estrategia de crecimiento interno [22], [23]. Otras entidades de origen provincial, como la Caja de Ahorros de Almería, pasa a formar parte de un grupo regional, Unicaja, con sede en Málaga, perdiéndose así parte del arraigo que se le tenía al Monte de Piedad.

En esta fase, Cajamar Caja Rural adquiere una dimensión nacional, al tiempo que adquiere una fortaleza impensable para una cooperativa de crédito, con cuotas de mercado en depósitos superiores al 50\%, que hoy por hoy, aún perduran.

Desde el punto de vista técnico, es en esta fase cuando comienzan las inversiones hacia nuevas estructuras de invernadero, más eficientes y productivas, como los invernaderos asimétricos [18]. En definitiva, se trata de dar respuesta a la demanda proveniente del mercado único europeo, por lo que se requería incrementar la productividad, así como la gama y variedad de los productos. 
En la tabla I, se observan las cuotas de mercado, tanto en créditos como en depósitos a otros sectores residentes, en la provincia de Almería y en España. En este sentido, resulta característico que el sector de cooperativas de crédito sólo representa el 6\% y el 7\% de los créditos y depósitos, respectivamente, a escala

entidad (enero 2013).

\begin{tabular}{|r|c|c|c|c|}
\hline Media española & \multicolumn{2}{|c|}{ Créditos sobre clientes } & \multicolumn{2}{c|}{ Depósitos de clientes } \\
& (otros sectores residentes) & (otros sectores residentes) \\
\cline { 2 - 5 } & Importe & $\%$ & Importe & $\%$ \\
\hline Bancos & 754.397 & $52 \%$ & 572.545 & $49 \%$ \\
\hline Cajas Ahorros & 615.179 & $42 \%$ & 510.600 & $44 \%$ \\
\hline Coop. Crédito & 89.806 & $6 \%$ & 85.679 & $7 \%$ \\
\hline Total Sector & $\mathbf{1 . 4 5 9 . 3 8 2}$ & $\mathbf{1 0 0} \%$ & $\mathbf{1 . 1 6 8 . 8 2 4}$ & $\mathbf{1 0 0} \%$ \\
\hline
\end{tabular}

\begin{tabular}{|r|c|c|c|c|}
\hline \multirow{2}{*}{ Almeria } & \multicolumn{2}{|c|}{ Créditos sobre clientes } & \multicolumn{2}{c|}{ Depósitos de clientes } \\
& (otros sectores residentes) & (otros sectores residentes) \\
\cline { 2 - 5 } & Importe & $\%$ & Importe & $\%$ \\
\hline Bancos & 6.058 & $30 \%$ & 1.616 & $21 \%$ \\
\hline Cajas Ahorros & 7.099 & $35 \%$ & 2.202 & $28 \%$ \\
\hline Coop. Crédito & 7.192 & $35 \%$ & 3.924 & $51 \%$ \\
\hline Total Sector & $\mathbf{2 0 . 3 4 9}$ & $\mathbf{1 0 0} \%$ & $\mathbf{7 . 7 4 2}$ & $\mathbf{1 0 0} \%$ \\
\hline
\end{tabular}

Asimismo, las cuotas provinciales indican la enorme descompensación que existe entre la captación de depósitos y la inversión crediticia, en lo que respecta a otros sectores residentes (7.742 millones de euros en depósitos a otros sectores residentes, frente a los 20.349 millones invertidos en forma de créditos). A pesar de ello, el arraigo de la única cooperativa de crédito almeriense, Cajamar Caja Rural, consigue captar uno de cada dos euros que el sector privado mantiene depositados en la provincia de Almería.

- Fase de reformulación del modelo (a partir de 2012). En esta etapa el modelo de desarrollo local basado en la intensidad en la producción agrícola no será suficiente para garantizar la supervivencia futura del sector, ni podrá absorber la oferta de mano de obra cualificada que genera la provincia. Es el momento de desarrollar nacional, mientras que en el caso de Almería los porcentajes son bien distintos. Así, el carácter marginal y poco representativo que se le puede atribuir al crédito cooperativo a escala nacional, no corresponde con la visión provincial. definitivamente una verdadera industria auxiliar para el campo almeriense, crear un entramado empresarial que tutele la investigación fitosanitaria $\mathrm{y}$, lo más importante, se aúnen los esfuerzos de cara a concentrar y planificar la oferta comercial.

Desde el punto de vista financiero, serán necesarias importantes inversiones que serán suministrados por la entidad cooperativa líder en España, con sede social en Almería, la ganancia de dimensión del sector agroalimentario facilitará la apelación directa a los mercados financieros.

\section{Conclusiones} Enero - Junio 2017 E-ISSN 2422-5053

El modelo de desarrollo local agroalimentario que se ha desarrollado en el sureste español ha basado su éxito en la explotación minifundista, de 2 hectáreas de media, de 
tipo familiar, dirigida por un agricultor que cotiza, normalmente, en el régimen especial de trabajadores autónomos. Dicho modelo productivo ha conseguido altas cotas de competitividad internacional. Esta particularidad del modelo almeriense aporta importantes ventajas para determinadas regiones, que basan su desarrollo en la agricultura. En el caso de Almería, el éxito del modelo se ha sido refrendado durante décadas.

Sin embargo, no parece adecuado abogar por una trasposición milimétrica del modelo almeriense sin valorar el perfil del territorio candidato, es decir: la existencia y el tipo de infraestructuras físicas, privadas y públicas que existe, la capacidad de consumo de la población autóctona, la proximidad a importantes mercados internacionales a los que derivar su producción vía exportaciones, etc. Es un modelo endógeno, fruto de la movilización de unos recursos productivos muy específicos, que han sido apoyados por una importante baza: la consolidación de un gran mercado próximo, estable y solvente, es decir, la Unión Europea.

Es por ello que, la adaptación de las principales características del modelo de desarrollo local agroindustrial almeriense a países sin transición industrial, con una incipiente actividad agrícola y capital humano para emplearlo en dichas tareas, puede ser una alternativa seria y digna al desarrollo industrial en sentido estricto.

Adicionalmente, hay que tener presente que el éxito de este modelo productivo está altamente correlacionado con la propia maduración del sector financiero, es decir, del sector de cooperativas de crédito, que en el caso de Almería se corresponde con Cajamar Caja Rural. Esta relación es la que explica las altas cotas de mercado en depósitos y en créditos sobre otros sectores residentes, superiores al 50\% y al 35\% respectivamente.
No existe ninguna cooperativa de crédito, ya sea caja laboral o caja rural, que ostente unas cotas similares, superando al resto de intermediarios.

Con respecto a la posibilidad de que el modelo de integración de Cajamar pueda ser un referente para que en países como Colombia se puedan desarrollar modelos de integración de sistemas financieros y sector productivo real lo que conllevaría la generación entre otras de las siguientes externalidades:

- Generar estructuras de concentración entre pequeñas cooperativas de crédito, sean estas multiactivas o especializadas [25].

- Propiciar incentivos de políticas públicas para la financiación de conglomerados agroindustriales en zonas de postconflicto [26].

- Propiciar acciones de cooperación técnica para la transferencia de conocimientos con movimientos agroalimentarios basados en organizaciones cooperativas como las de Almería, Israel y Turquía.

- Realizar acciones de acondicionamiento de los territorios de postconflicto para la reorientación económica basada en la consolidación de condiciones cívicas para la cooperación, la potenciación de emprendimientos agrarios y la estimulación de actuación en red $\mathrm{y}$ circuitos económicos [26].

- El grado de vinculación de esta cooperativa de crédito con el sector agrícola español es lo que podría explicar una situación excepcional: la de la cooperativa de crédito más importante del país y el sector de agrícola más productivo y con mayor orientación exterior de España. 


\section{Referencias}

[1] J. Martinez-Paz, F. Martinez-Carrasco and M. Dios-Palomares, "Analysis of the Evolution of Protected Horticulture in Almeria Area: Cycles, Structure an Growth", Acta Horticulture, vol. 559, pp. 713-718, 2001.

[2] F.J. Ferraro, El sistema productivo almeriense y los condicionamientos hidrológicos, Madrid: Cívitas, 2000.

[3] F. González Olivares y J.J. González Rodriguez, "Almería: el milagro de una agricultura intensiva", Papeles de Economía Española, $\mathrm{n}^{\mathrm{o}} 16$, pp. 152168, 1983.

[4] A. Tolón Becerra y X. Lastra Bravo, "La agricultura intensiva del poniente almeriense. Diagnóstico e instrumentos de gestión ambiental", Revista Electrónica de Medio Ambiente, $\mathrm{n}^{\mathrm{o}} 8$, pp. 18-40, 2010.

[5] J.A. Gómez-Limón, J. Calatrava, A. Garrido, F. J. Sáez y À. Xabadia, La economía del agua de riego en España. Una perspectiva regional, Colección Economía, Almería, España.: Fundación Cajamar, 2009.

[6] I. Ruiz Guerra, V. M. Martín López and V. Molina Moreno, "Olive Oil intangibility for competitive advantage", Intangible Capital, vol. 8, nº 1, pp. 150-179, 2012.

[7] Instituto Nacional de Estadística, "Contabilidad Regional de España. Base 2010", 19 Diciembre 2014. [En línea]. Disponible en: http://www.ine. es/prensa/np888.pdf.

[8] Servicio Oficial de Inspeccion, Vigilancia y Revision de Exportaciones, "Estadísticas de Comercio Mundial", ICEX, Madrid, 2000.
[9] A. Marshall, Industry and Trade, London: Macmillan, 1920.

[10] F.J. Cortés García y L.J. Belmonte Ureña, "La base social de las cooperativas de crédito. La importancia de la responsabilidad social corporativa", Revista de Estudios Empresariales. Segunda época, n 2, pp. 35-53, 2010.

[11] L.J. Belmonte Ureña, "El nuevo modelo de negocio de las cooperativas de crédito españolas. Perspectivas futuras", Papeles de Economía Española, n ${ }^{\circ} 130$, pp. 244-257, 2011.

[12] F.J.CortésGarcía, "Elmodeloeconómico almeriense y su financiación”, Boletín Económico de ICE, no 2728, pp. 17-25, 2002.

[13] J.C. Pérez Mesa, "El sector hortofrutícola almeriense frente a los cambios de la oferta y la demanda internacional. En: Informe Anual del Sector Agrario en Andalucía 2008", Analistas Económicos de Andalucía Unicaja, Málaga, España., 2008.

[14] I. Ruiz Guerra and V.M. Martín López, "Strategic Management for the Internationalization and Cooperative Markets", Procedia Social and Behavioral Sciences, $\mathrm{n}^{\circ} 24$, p. 769-780, 2011.

[15] G.M. Marin, R. Peral y M.A. Bonillo, "Oportunidades en los mercados exteriores para la industria auxiliar de la agricultura intensiva", Boletín Económico de ICE, n ${ }^{\mathrm{o}} 2722$, pp. 31-41, 2002.

[16] Fundación Cotec, "Documento sobre oportunidades tecnológicas. Invernaderos bajo plástico", Fundación Cotec, Madrid, 2009.
Enero - Junio 2017 ISSN 0122-820X E-ISSN 2422-5053 PP: 48-58 
No. 1

Enero - Junio 2017

ISSN 0122-820X

E-ISSN 2422-5053

PP: 48-58
[17] D.L. Valera, A.A. Peña, J. Perez, A.J. Álvarez y F.D. Molina, "Tensoestructuras como sistemas de protección de cultivos: el invernadero Tipo Almería", de Control climático en invernaderos, Almeria, España, Servicio de Publicaciones de la Universidad de Almería, 2003.

[18] F. Camacho, Técnicas de producción de frutas y hortalizas en los cultivos protegidos del sureste español, Almería, España: Caja Rural de Almería, 1999.

[19] Banco de España, "Boletín Estadístico del Banco de España, 1987-2012.," 2012. [En línea]. Disponible en: http:// www.bde.es/webbde/es/estadis/infoest/ bolest $4 . h$ tml.

[20] J. Rivera, "La política de colonización agraria en el Campo de Dalias (1940-1990)", Instituto de Estudios Almerienses, Almería, España, 2000.

[21] J.A. Aznar J.A. y A. Sánchez, "Innovación y distrito en torno a un "milagro": la configuración del sistema productivo local de la agricultura intensiva en Almería",Revista de Historia Industrial, vol. 19, $\mathrm{n}^{\circ} 42$, pp. 157-193, 2010.

[22] A. Melian, "Expansión y crecimiento de las cooperativas de crédito durante el periodo 1993-2007 en la Comunidad Valenciana", REVESCO. Revista de
Estudios Cooperativos, $\mathrm{n}^{\circ}$ 98, pp. 97117, 2009.

[23] L.J.Belmonte, "Elsectorde cooperativas de crédito en España. Un estudio por Comunidades Autónomas", Consejo Económico y Social de Andalucía Colección Premio de Investigación, Sevilla, España, 2007.

[24] Asociacion Española de Banca- AEB, "Distribución provincial de empleados, crédito y depósitos de la clientela (12013)", Informe de la Secretaría General AEB", Asociacion Española de BancaAEB, Madrid, España, 2013.

[25] L. Belmonte, J. Álvarez y J. Álvarez, "La estrategia de concentración de las cooperativas de crédito españolas. Análisis de los sistemas institucionales de protección" Revista Finanzas y Política Económica, vol 6, no. 2, 2013.

[26] J. Álvarez, H. Zabala, A. García y C. Orrego, Crescencio, Marco para el fomento de la economía solidaria en territorios rurales de Colombia. Centro de Investigación, Documentación e Información de la Economía Social, Pública y Cooperativa - CIRIEC, Bogotá, 2015. 